\title{
Improved 1-h rapid immunostaining method using intermittent microwave irradiation: practicability based on 5 years application in Toyama Medical and Pharmaceutical University Hospital
}

\author{
Tokimasa Kumada, Koichi Tsuneyama, Hideki Hatta, Shin Ishizawa and Yasuo Takano \\ Department of Pathology, Faculty of Medicine, Toyama Medical and Pharmaceutical University, Sugitani, \\ Toyama, Japan
}

\begin{abstract}
Immunostaining depending on antigen-antibody specificity is the commonest approach for determining the localization of specific antigens in tissue sections. This procedure is applicable not only with frozen or specially fixed samples, but also has proved reliable with formalin-fixed paraffin-embedded tissue sections through improvement of antigen-retrieval. Immunostaining is thus firmly established as a tool for diagnostic pathology and in our institute multiple antibodies are applied for $13-15 \%$ of the cases examined, as well as H\&E staining. With the standard approach, approximately $3 \mathrm{~h}$ is necessary from the beginning of deparaffinization till covering sections with the Envision system. We utilized intermittent microwave irradiation for 10 min during hybridization with primary and secondary antibodies in a special moist-chamber, to achieve all immunostaining steps within $1 \mathrm{~h}$ in 178 primary antibodies frequently used for diagnostic pathology. According to our 5 years experience, such microwave irradiation not only obtained significant specific staining for enhancing the specificity of antigen-antibody reactions, but also inhibited nonspecific binding. We present herein the details of the methodology and recommendations for its application with particular primary antibodies. This method can contribute to savings in time and energy, allowing pathologists to rapidly obtain diagnostic information. Modern Pathology (2004) 17, 1141-1149, advance online publication, 28 May 2004; doi:10.1038/modpathol.3800165
\end{abstract}

Keywords: AML; $t(8 ; 21)$; quantitative immunophenotype

Immunostaining depending on antigen-antibody specificity is the commonest approach adopted to detect specific antigens in tissue sections. ${ }^{1-3}$ Recent innovations in antigen-retrieval procedures for formalin-fixed paraffin embedded tissue sections have brought increased reliability to immunostaining of routinely produced paraffin-embedded tissue sections. ${ }^{4-9}$ This is now an established diagnostic tool for general use in the histopathology laboratory and in our institute, Toyama Medical and Pharmaceutical University Hospital, 486 out of 3854 cases (13\%) examined in 2002 required immunostaining in addition to routine H\&E staining. Because around 10 antibodies were applied per case on average,

Correspondence: Dr K Tsuneyama, MD, PhD, Department of Pathology, Faculty of Medicine, Toyama Medical and Pharmaceutical University, 2630 Sugitani, Toyama 930-0194, Japan.

E-mail: ktsune@ms.toyama-mpu.ac.jp

Received 03 October 2003; revised 20 December 2003; accepted 22 December 2003; published online 28 May 2004 almost 5000 immunostained specimens were produced to assist in diagnosis.

The standard immunostaining procedure with the Envision system (DAKO, Carpinteria, CA, USA) takes around $3 \mathrm{~h}$ from beginning of deparaffinization of the specimens till coverslipping. In many laboratories, overnight incubation with the primary or secondary antibodies is often employed, to fit with business hours, but this precludes same day service. To facilitate rapid pathological diagnosis, we have developed an immunostaining method utilizing intermittent microwave irradiation during incubation with antibodies inside a specially designed moist chamber. By this method, we can complete all steps for immunostaining within $1 \mathrm{~h}$, at the same time maintaining excellent quality. This immunostaining approach (rapid method) has now been used as the routine procedure for 5 years in our hospital. In the present report, we described detailed directions and efficacy of this method with concrete examples. Additionally, accumulated information 
on 178 primary antibodies used for diagnosis applying this method is provided.

\section{Materials and methods}

\section{1-h Immunostaining Procedure (Table 1)}

\section{Tissue preparation}

Tissue sections cut at $4 \mu \mathrm{m}$ from representative tissue blocks are prepared and placed in a paraffin-oven at $60^{\circ} \mathrm{C}$ to remove most of the paraffin.

\section{Deparaffinization (5 min)}

For complete deparaffinization, specimens are then passed through xylene and a series of alcohol dilutions. Because the amount of paraffin is markedly reduced by oven heating, a total of $5 \mathrm{~min}$ is sufficient for this process. After soaking the specimens in the last jar with $50 \%$ alcohol, specimens are rinsed well under running water for $2 \mathrm{~min}$.

\section{Antigen-retrieval (10 min)}

For the antigen retrieval, we use either standard microwave treatment or enzyme treatment. ${ }^{9-13}$ For the enzyme digestion, specimens are soaked in enzyme solution for $6 \mathrm{~min}$ at $37^{\circ} \mathrm{C}$. For microwave pretreatment, $10 \mathrm{~min}$ is necessary. A pressure cooker for home cooking use (Type RC 11, Asahi Light Metal Industry, Osaka, Japan) and standard microwave equipment (maximum $500 \mathrm{~W}$, type RE-11, Sharp, Tokyo, Japan) are suitable for this irradiation step. ${ }^{14,15}$ At the step of deparaffinization, the nonmetal containing plastic-made pressure cooker with half-filled target retrieval buffered solution (TRS; DAKO) is beforehand irradiated to cause boiling in a microwave oven for 5 min (Figure 1a). Deparaffinized specimens are then soaked in boiled TRS and irradiated for $10 \mathrm{~min}$ more under high pressure. After irradiation, specimens are taken from the boiling TRS and rinsed well under running water for $2 \mathrm{~min}$.

Table 1 1-h Immunostaining procedure

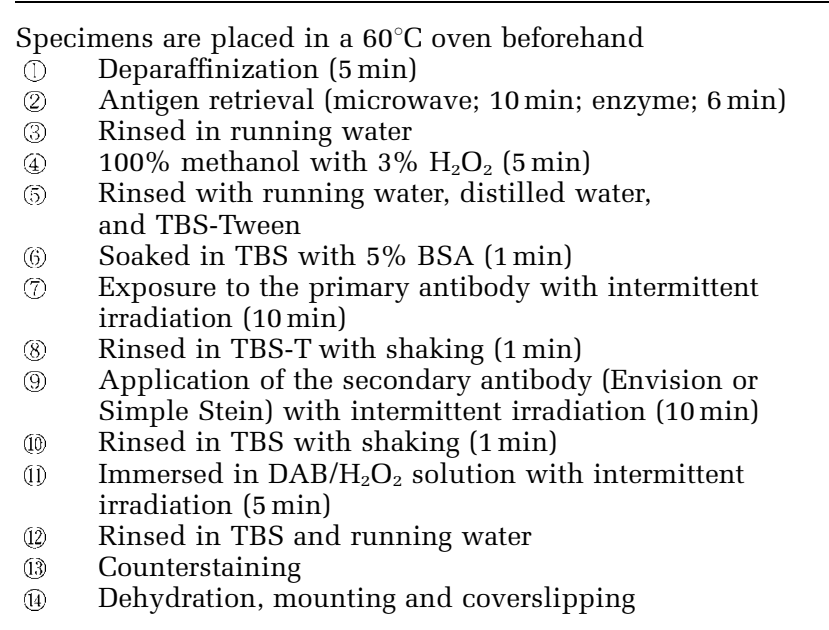

Blockage of endogenous peroxidase (5 min)

After soaking in the methanol solution with $3 \%$ $\mathrm{H}_{2} \mathrm{O}_{2}$ for $5 \mathrm{~min}$, specimens are rinsed well under running water for $2 \mathrm{~min}$ and then Tris-buffered saline (TBS) containing 1\% Tween for $1 \mathrm{~min}$.

\section{Blocking nonspecific antigen-antibody reaction (1 min)}

To prevent nonspecific antibody reactions, specimens are immersed in 5\% bovine serum albumin (BSA, Sigma, Steinheim, Germany) for 1 min. This step can be omitted because primary antibodies are already diluted with 5\% BSA.

\section{Primary antibody reaction (10 $\mathrm{min}$ )}

Primary antibodies are diluted with 5\% BSA to their optimal dilutions and applied directly to the specimens in plastic moist chambers (Figure 1b), which are then placed inside the special microwave equipment (MI-77, Azumaya, Tokyo, Japan), and irradiated intermittently (250 W, 4-s-on and 3-s-off) avoiding excessive temperature increase. ${ }^{16}$ We preliminary compared the specificity and intensity of the target staining in various incubation time, of which was 5, 10 and $30 \mathrm{~min}$, respectively with intermittent microwave irradiation. The result of that in $10 \mathrm{~min}$ was ensured the sufficient staining quality shown in Figure 2. The temperature of the liquid on the slide was just increased within $2-3^{\circ}$ during $10 \mathrm{~min}$ with intermittent microwave irradiation in 250W. A maximum of six moist chambers, each carrying 10 specimens (maximum of 60 specimens), can be placed inside the microwave equipment. Microwave irradiation is effected evenly in each moist chamber (Figure 1c). After irradiation, specimens are washed with TBS-Tween buffer in prepared bottles (Figure 1d). Specimens are shaken several times in one bottle and then moved to the next one and then the next. A total of 1 min of washing is sufficient to remove the excess primary antibodies from the specimens. This was preliminary confirmed by comparing the staining quality using specimens with no washing, the present system and full time $(5 \min \times 3)$ washing (Figure 3$)$.

\section{Secondary antibody reaction (10 $\mathrm{min})$}

For the secondary antibody reaction, peroxidaseconjugated Envision kit (Envision-PO, Envision System; DAKO, Carpinteria, CA, USA) for mouse or rabbit primary antibodies or peroxidase-conjugated Histofine-Simplestain kit (Simplestain MAXPO, Nichirei, Tokyo, Japan) for goat primary antibodies are applied on the specimens in the moist chambers. Irradiation is then performed intermittently for $10 \mathrm{~min}$, as described above, followed by washing with TBS for 10-12 s with shaking 5 times (total $1 \mathrm{~min}$ ). 
Color development with substrate and counterstaining (5 min)

Specimens are immersed in freshly prepared DAB (Sigma) solution with $\mathrm{H}_{2} \mathrm{O}_{2}$ in a glass jar and directly
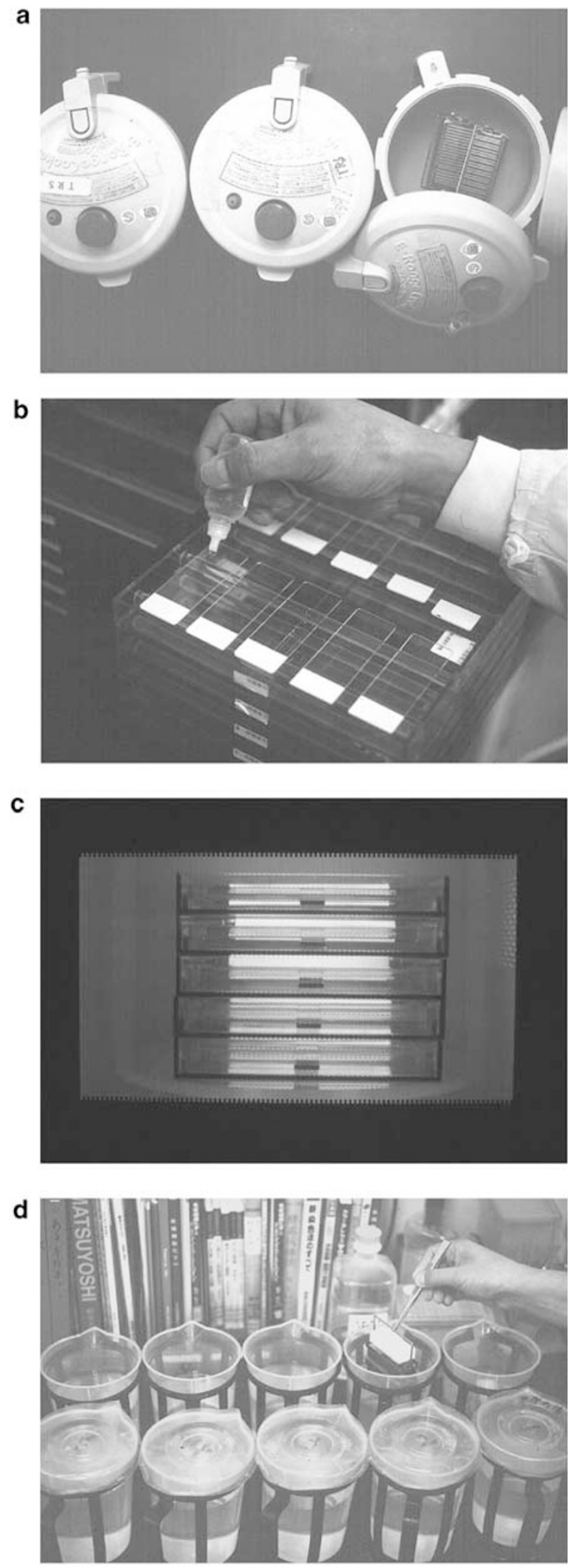

irradiated intermittently for 5 min inside the microwave equipment. After recognition of positive staining under the light microscope, specimens are rinsed well under running water for $2 \mathrm{~min}$, then counterstained with Meyer's hematoxylin and coverslipped for microscopic examination.

Primary antibodies applied with the rapid immunostaining method

After 1998, on average, about 450-500 cases per year required additional immunostaining for diagnosis, around 10 antibodies per case. Almost all immunostaining was performed using our rapid method. We now have experience with a total of 180 primary antibodies. All have been compared with both standard and rapid protocols staining with regard to sensitivity and specificity. Optimal dilutions and methods for antigen retrieval have been determined for each, with review of quality by both staining technicians and diagnostic pathologists, allowing choice of the most suitable methodology. Accumulated information for 180 primary antibodies is listed in Table 2.

\section{Results}

\section{Efficacy of Our Modified Rapid Immunostaining Procedure}

Time period

With our rapid method, all steps of immunostaining can be completed within $1 \mathrm{~h}$. Although this depends on the number of the specimens, we succeeded in finishing the whole immunostaining within $1 \mathrm{~h}$ in more than $90 \%$ cases for which it was applied in the last year. In contrast, with the standard immunohistochemical method, at least $3 \mathrm{~h}$ are required to complete the staining steps, because of the hour incubation periods for both primary and secondary antibodies.

\section{Staining quality}

To evaluate the staining quality of our quick method, we compared the staining sensitivity, intensity and specificity with 180 primary antibodies frequently used for assistance to diagnosis. In all cases (100\%), the rapid method provided equal or better quality of immunostaining than the standard method. Staining intensity was almost the same with both methods, while nonspecific background staining was less

Figure 1 (a) Antigen retrieval was carried out in a special pressure cooker with target retrieval buffered solution. (b) Specimens were incubated inside plastic moist chambers, suitably sized for the microwave equipment. (c) Six moist chambers were stacked inside the special microwave equipment. Fluorescence lamps inside the moist chambers provide luminescence during the irradiation. (d) For rapid washing, five large bottles filled with buffered solution are prepared. 

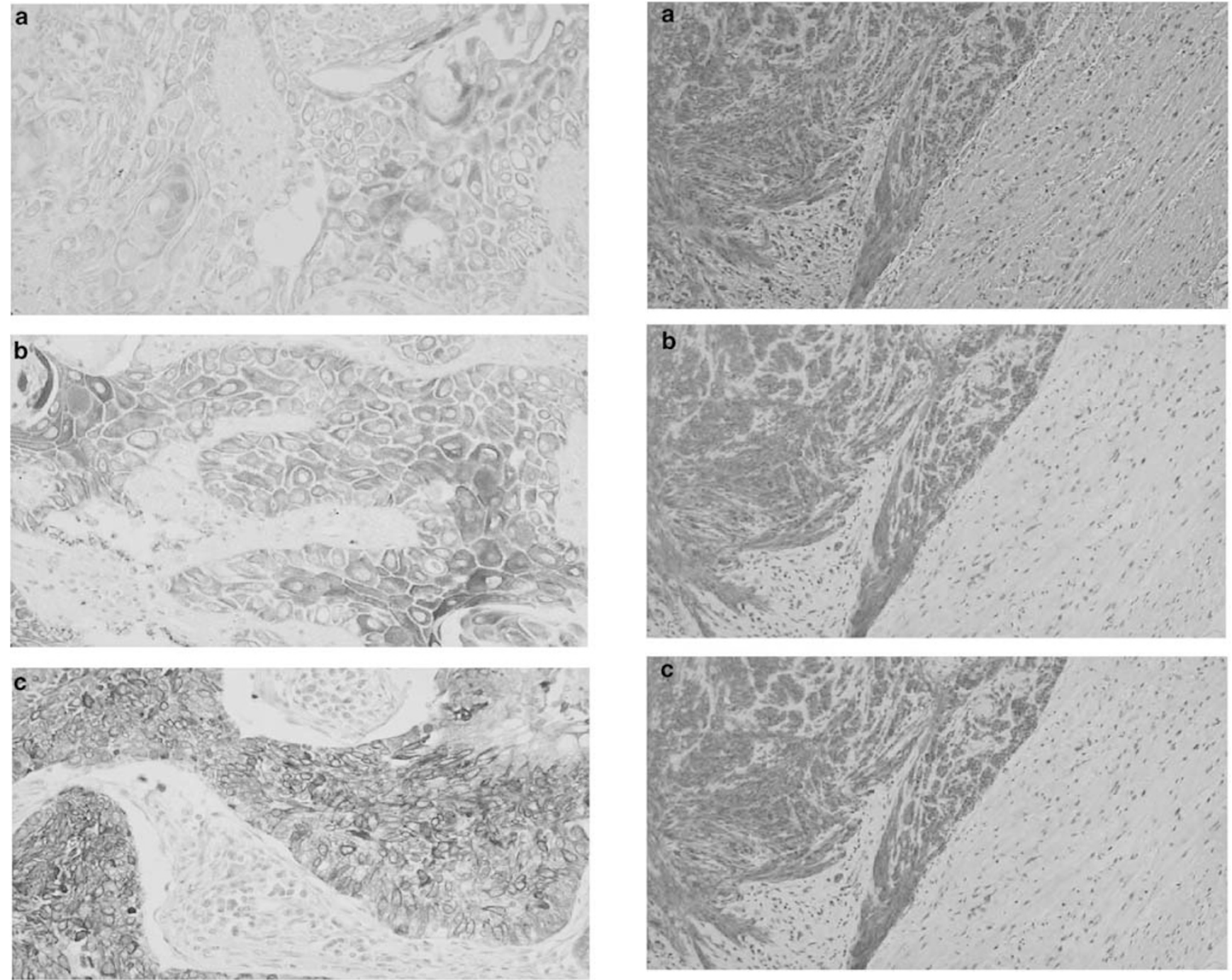

Figure 2 (a)-(c) Specimens with squamous cell carcinoma incubated with an anti-cytokeratin $5 / 6$ for (a) $5 \mathrm{~min}$, (b) $10 \mathrm{~min}$ and (c) $30 \mathrm{~min}$ intermittent microwave irradiation. (a) Weak positive staining was observed sparsely, (b) diffuse positive staining was observed in almost all cancer cells, (c) diffuse and intense staining was observed in almost all cancer cells. Evaluation of specific positive reaction could be performed correctly in the specimen with 10 min irradiation.

pronounced with the rapid method (Figure 4). In the case of leukemia cells in the liver, our immunohistochemical analysis succeeded in demonstrating the same results as flow-cytometry with regard to surface marker profiles. Even though overnight incubation was recommended in data sheets, the rapid method often worked well with high titers.

\section{Optimal staining conditions for 180 primary antibodies}

During our 5 years experience of the rapid immunostaining method for the routine diagnostic use, we have examined suitable staining conditions for 180 primary antibodies. With most of the antibodies examined, the recommended dilution titer from the

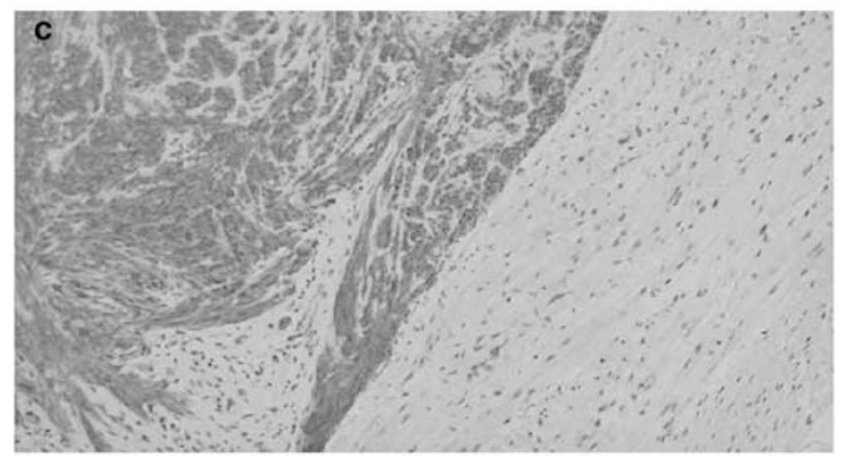

Figure 3 (a)-(c) c-kit immunostaining of a gastrointestinal stromal tumor with different washing procedures. (a) No washing after primary antibody incubation, (b) our quick washing method for $1 \mathrm{~min}$, (c) recommended washing for $5 \mathrm{~min}$, three times. Note background nonspecific staining was in (a), but not (b) and (c).

data sheet was first tried with the rapid method. Three different dilution titers of 1:50, 1:100 and 1:200 were employed if there was no company recommendation. In all, 30 primary antibodies were directly applied without dilution as recommended by the manufacturer. With all antibodies except that for calretinin, the concentration was sufficient for significant positive staining with our quick method. Basing on the company recommendation of overnight incubation for calretinin, the concentration was increased, and significant positive staining resulted with the rapid protocol.

For antigen retrieval, 49 out of 178 (27.5\%) antibodies required protease $\mathrm{K}$ pretreatment for $6 \mathrm{~min}$ at $37^{\circ} \mathrm{C}$, and the other 129 antibodies $(72.5 \%)$ required microwave pretreatment for $10 \mathrm{~min}$. Among those 129 antibodies, six (4.7\%) required EDTA solution and one $(0.8 \%)$ required 
Table 2 List of antibodies and staining dilutions for the rapid method

\begin{tabular}{|c|c|c|c|c|c|}
\hline Antibodies & Source & Pretreatment & Supplier & Optimal dilution & Data sheet \\
\hline AE1/AE3 & M & $\operatorname{Pr}$ & Boehringer & 200 & $100-300$ \\
\hline AE1 & $\mathrm{M}$ & $\operatorname{Pr}$ & Boehringer & 600 & $100-300$ \\
\hline AE3 & $\mathrm{M}$ & $\operatorname{Pr}$ & Boehringer & 100 & ND \\
\hline AFP & $\mathrm{P}$ & & Immunon & 100 & $50-100$ \\
\hline ACTH & $\mathrm{P}$ & & DAKO & Neat & Neat \\
\hline Amyloid A & $\mathrm{M}$ & $\operatorname{Pr}$ & DAKO & 100 & $50-100$ \\
\hline Amyloid P & $\mathrm{P}$ & & DAKO & 200 & $200-300$ \\
\hline Albumin & $\mathrm{P}$ & & DAKO & 500 & ND \\
\hline$\alpha 1-\mathrm{ACT}$ & $\mathrm{P}$ & & Immunon & 100 & $50-100$ \\
\hline$\alpha 1-\mathrm{AT}$ & $\mathrm{P}$ & & Immunon & 100 & $50-100$ \\
\hline ALK (p80) & M & & DAKO & 25 & $25-50$ \\
\hline bcl-1 (Cyclin D1) & $\mathrm{M}$ & & IBL & 100 & $20-50$ \\
\hline bcl-2 & $\mathrm{M}$ & & DAKO & 40 & $40-80$ \\
\hline GCDFP-15 & M & & Signet & 50 & $10-60$ \\
\hline BRST-3 (B72.3) & $\mathrm{M}$ & & Signet & 50 & $10-50$ \\
\hline BerEP4 & M & $\operatorname{Pr}$ & DAKO & 80 & $50-100$ \\
\hline BCG & $\mathrm{P}$ & & DAKO & 3000 & 1000 \\
\hline$\beta 2$ microgloblin & $\mathrm{P}$ & & DAKO & 500 & 500 \\
\hline CD1a (Leu-6) & $\mathrm{M}$ & & Immunontech & Neat & Neat \\
\hline CD3 (mono) & $\mathrm{M}$ & & Immunontech & Neat & Neat \\
\hline CD3 (poly) & $\mathrm{P}$ & $\operatorname{Pr}$ & DAKO & 50 & $50-100$ \\
\hline CD4 & $\mathrm{M}$ & & NOVO & 10 & $20-40$ \\
\hline CD5 & $\mathrm{M}$ & & NOVO & 50 & $25-50$ \\
\hline CD8 & $\mathrm{M}$ & & DAKO & 25 & $50-100$ \\
\hline CD10 & $\mathrm{M}$ & & NOVO & 40 & $50-100$ \\
\hline CD15(Leu-M1) & M & $\operatorname{Pr}$ & Becton Dickinson & 20 & ND \\
\hline CD20 (L-26) & $\mathrm{M}$ & & DAKO & 100 & $50-100$ \\
\hline CD21 & $\mathrm{M}$ & $\operatorname{Pr}$ & DAKO & 20 & $10-20$ \\
\hline CD23 & $\mathrm{M}$ & & NOVO & 20 & $20-40$ \\
\hline CD30 (ki-1) & $\mathrm{M}$ & $\operatorname{Pr}$ & Immunontech & Neat & Neat \\
\hline CD31 & M & & NOVO & 100 & $50-100$ \\
\hline CD34 & $\mathrm{M}$ & $\operatorname{Pr}$ & NOVO & 50 & 50 \\
\hline CD35 & M & $\operatorname{Pr}$ & DAKO & 20 & $10-20$ \\
\hline CD38 & $\mathrm{M}$ & $\mathrm{E}$ & NOVO & 100 & $100-200$ \\
\hline CD41 & $\mathrm{M}$ & $\operatorname{Pr}$ & DAKO & 100 & $50-100$ \\
\hline CD43 (MT-1) & M & & Bio Science & 40 & $40-50$ \\
\hline CD44 & $\mathrm{M}$ & & DAKO & 50 & $40-80$ \\
\hline CD45RO (UCHL1) & M & & DAKO & 50 & $25-50$ \\
\hline CD56 (N-CAM) & $\mathrm{M}$ & & NOVO & 50 & $50-100$ \\
\hline CD57 (Leu-7) & $\mathrm{M}$ & & Becton Dickinson & 20 & $20-50$ \\
\hline CD61 & M & $\operatorname{Pr}$ & DAKO & 100 & $50-200$ \\
\hline CD68 (KP-1) & $\mathrm{M}$ & $\operatorname{Pr}$ & DAKO & 100 & $50-100$ \\
\hline CD68 (PG-M1) & M & $\operatorname{Pr}$ & DAKO & 50 & $50-100$ \\
\hline CD79a & $\mathrm{M}$ & & Immunontech & 50 & 50 \\
\hline CD83 & $\mathrm{M}$ & & NOVO & 40 & $20-40$ \\
\hline CD138 & M & & DAKO & 25 & $25-50$ \\
\hline CAK1 & $\mathrm{M}$ & Ur & Signet & 40 & 40 \\
\hline CA15-3 & M & & TFB & 50 & 50 \\
\hline CA19-9 & $\mathrm{M}$ & & Japan Tanner & 20 & ND \\
\hline CA125 & M & & Japan Tanner & 50 & ND \\
\hline Calcitonin & $\mathrm{P}$ & & DAKO & Neat & Neat \\
\hline CAM5.2 & $\mathrm{M}$ & $\operatorname{Pr}$ & Becton Dickinson & Neat & Neat \\
\hline Calretinin & $\mathrm{P}$ & & SWANT & 500 & $1000-5000$ \\
\hline C3c & $\mathrm{P}$ & $\operatorname{Pr}$ & DAKO & 200 & $50-100$ \\
\hline CEA & $\mathrm{M}$ & & DAKO & 50 & $25-50$ \\
\hline Chromogranin A & $\mathrm{P}$ & & DAKO & 100 & $50-150$ \\
\hline c-kit & $\mathrm{P}$ & & IBL & 20 & $5-10$ \\
\hline Cytokeratin5/6 & M & $\operatorname{Pr}$ & Boehringer & 50 & $50-100$ \\
\hline Cytokeratin 7 & M & $\operatorname{Pr}$ & Bio Genex & 100 & $100-200$ \\
\hline Cytokeratin 8/18 & M & $\operatorname{Pr}$ & NOVO & 40 & 40 \\
\hline Cytokeratin 14 & $\mathrm{M}$ & $\mathrm{H}$ & Bio Genex & 50 & $30-60$ \\
\hline Cytokeratin 20 & $\mathrm{M}$ & $\operatorname{Pr}$ & DAKO & 50 & $20-40$ \\
\hline СК34 $\beta$ E12 & M & $\operatorname{Pr}$ & DAKO & 50 & $25-75$ \\
\hline CK LP34 & $\mathrm{M}$ & $\operatorname{Pr}$ & DAKO & 100 & $50-100$ \\
\hline Collagen 1 & G & $\operatorname{Pr}$ & Japan Tanner & 100 & $100-200$ \\
\hline Collagen 3 & G & $\operatorname{Pr}$ & Japan Tanner & 400 & 400 \\
\hline Collagen 4 & M & $\operatorname{Pr}$ & DAKO & 100 & $50-100$ \\
\hline
\end{tabular}


Table 2 Continued

\begin{tabular}{|c|c|c|c|c|c|}
\hline Antibodies & Source & Pretreatment & Supplier & Optimal dilution & Data sheet \\
\hline Collagen 5 & G & $\operatorname{Pr}$ & Japan Tanner & 300 & 300 \\
\hline Collagen 6 & G & $\operatorname{Pr}$ & Japan Tanner & 20 & $20-40$ \\
\hline CMV & $\mathrm{M}$ & $\operatorname{Pr}$ & DAKO & 25 & $25-30$ \\
\hline C-erbB2 & $\mathrm{M}$ & & NOVO & 40 & 40 \\
\hline СРР32 & $\mathrm{P}$ & & DAKO & 250 & 250 \\
\hline Desmin & $\mathrm{M}$ & & DAKO & 100 & $50-100$ \\
\hline EBV (LMP-1) & $\mathrm{M}$ & $\operatorname{Pr}$ & DAKO & 25 & $25-50$ \\
\hline EBV (ZEBRA) & $\mathrm{M}$ & & DAKO & 25 & $20-40$ \\
\hline EG2 & $\mathrm{M}$ & & Pharmacia & 100 & ND \\
\hline EMA & $\mathrm{M}$ & & DAKO & 100 & $50-100$ \\
\hline Estrogen receptor & $\mathrm{M}$ & & Immunontech & 50 & 50 \\
\hline Fibrinogen & $\mathrm{P}$ & & DAKO & 500 & $100-500$ \\
\hline Fibronectin & $\mathrm{P}$ & $\operatorname{Pr}$ & DAKO & 500 & $200-400$ \\
\hline Factor VIII (vWF) & $\mathrm{P}$ & $\operatorname{Pr}$ & DAKO & 200 & 200 \\
\hline Ferritin & $\mathrm{P}$ & & DAKO & 100 & 100 \\
\hline FSH & $\mathrm{P}$ & & DAKO & Neat & Neat \\
\hline Fibroblast & $\mathrm{M}$ & & DAKO & 30 & $50-100$ \\
\hline Gastrin & $\mathrm{P}$ & & DAKO & Neat & Neat \\
\hline Glucagon & $\mathrm{P}$ & & DAKO & Neat & Neat \\
\hline $\mathrm{GH}$ & $\mathrm{P}$ & & DAKO & Neat & Neat \\
\hline G-CSF & $\mathrm{M}$ & & IBL & 100 & $\mathrm{ND}$ \\
\hline GFAP & $\mathrm{P}$ & & DAKO & Neat & Neat \\
\hline Granzyme B & $\mathrm{M}$ & & KAMIYA & 50 & 20 \\
\hline GTH receptor & $\mathrm{M}$ & & NOVO & 10 & $10-20$ \\
\hline Glycophrin A & $\mathrm{M}$ & & DAKO & Neat & Neat \\
\hline HBME1 & M & & DAKO & 50 & 50 \\
\hline h-Caldesmon & $\mathrm{M}$ & & DAKO & 100 & $50-100$ \\
\hline hCG & $\mathrm{P}$ & & DAKO & 500 & 200 \\
\hline hPL & $\mathrm{P}$ & & DAKO & Neat & Neat \\
\hline Hemoglobin & $\mathrm{P}$ & & DAKO & 100 & 100 \\
\hline H. pylori & $\mathrm{P}$ & $\operatorname{Pr}$ & DAKO & 50 & $50-80$ \\
\hline HHF35 actin & $\mathrm{M}$ & & Immunon & 50 & 50 \\
\hline HIV p24 & $\mathrm{M}$ & $\operatorname{Pr}$ & DAKO & 10 & $5-10$ \\
\hline HMB45 & $\mathrm{M}$ & & Bio Genex & 50 & $50-100$ \\
\hline HVS I & $\mathrm{P}$ & & Immunon & 100 & $50-100$ \\
\hline HVS II & $\mathrm{P}$ & & Immunon & 100 & $50-100$ \\
\hline HGM-45M1 & $\mathrm{M}$ & & NOVO & 50 & 50 \\
\hline HPV & $\mathrm{P}$ & & NICHIREI & Neat & Neat \\
\hline Hepatocyte & $\mathrm{M}$ & & DAKO & 25 & $25-50$ \\
\hline $\mathrm{HBc}$ & $\mathrm{P}$ & & DAKO & Neat & Neat \\
\hline HBs & M & & DAKO & Neat & Neat \\
\hline HHV 6 & $\mathrm{M}$ & & Biogenesis & 100 & $25-100$ \\
\hline $\operatorname{Ig} \mathrm{A}$ & $\mathrm{P}$ & $\operatorname{Pr}$ & Immunon & 200 & $50-100$ \\
\hline Ig D & $\mathrm{P}$ & $\operatorname{Pr}$ & DAKO & 200 & $50-200$ \\
\hline Ig E & $\mathrm{M}$ & $\operatorname{Pr}$ & DAKO & 100 & $50-100$ \\
\hline Ig $G$ & M & $\operatorname{Pr}$ & DAKO & 80 & $40-80$ \\
\hline Ig M & $\mathrm{M}$ & $\operatorname{Pr}$ & DAKO & 100 & $50-100$ \\
\hline Insulin & $\mathrm{P}$ & & DAKO & Neat & Neat \\
\hline Lactoferin & $\mathrm{P}$ & & DAKO & 100 & $100-150$ \\
\hline Laminin & $\mathrm{P}$ & $\operatorname{Pr}$ & HEYL & 100 & $100-250$ \\
\hline LCA & M & & DAKO & 200 & $50-200$ \\
\hline L-chain $\kappa$ & $\mathrm{M}$ & $\operatorname{Pr}$ & DAKO & 100 & $50-100$ \\
\hline L-chain $\lambda$ & $\mathrm{M}$ & $\operatorname{Pr}$ & DAKO & 400 & $200-400$ \\
\hline Lysozyme & $\mathrm{P}$ & $\operatorname{Pr}$ & DAKO & 200 & 200 \\
\hline LN 1 & $\mathrm{M}$ & & NICHIREI & Neat & Neat \\
\hline LN 2 & $\mathrm{M}$ & & NICHIREI & Neat & Neat \\
\hline LN 3 & $\mathrm{M}$ & & NICHIREI & Neat & Neat \\
\hline $\mathrm{LH}$ & $\mathrm{P}$ & & DAKO & Neat & Neat \\
\hline Mac 387 & $\mathrm{M}$ & $\operatorname{Pr}$ & DAKO & 100 & 100 \\
\hline Mucin (HIK 1083) & $\mathrm{M}$ & & KANTO Chemical & 30 & $10-50$ \\
\hline Muc-1 & $\mathrm{M}$ & & NOVO & 100 & 100 \\
\hline Muc-2 & $\mathrm{M}$ & & NOVO & 100 & $100-200$ \\
\hline MIC 2 & $\mathrm{M}$ & & DAKO & 50 & $50-75$ \\
\hline Myoglobin & $\mathrm{P}$ & & DAKO & 1000 & $100-200$ \\
\hline Myosin & $\mathrm{M}$ & & Immunon & 150 & 100-200 \\
\hline Metallottionein & M & & DAKO & 50 & 50 \\
\hline MIB-1 & M & & DAKO & 50 & 50 \\
\hline Melan A & $\mathrm{M}$ & & NOVO & 25 & $25-75$ \\
\hline MAST CELL & $\mathrm{M}$ & $\operatorname{Pr}$ & DAKO & 50 & $50-100$ \\
\hline
\end{tabular}


Table 2 Continued

\begin{tabular}{|c|c|c|c|c|c|}
\hline Antibodies & Source & Pretreatment & Supplier & Optimal dilution & Data sheet \\
\hline $\mathrm{MPO}$ & $\mathrm{P}$ & & DAKO & 100 & $100-200$ \\
\hline MBP & $\mathrm{P}$ & & DAKO & 100 & $100-150$ \\
\hline Neurofilament & $\mathrm{M}$ & & DAKO & 50 & $50-100$ \\
\hline Neut.Elastasa. & M & & DAKO & 100 & $50-150$ \\
\hline NSE & $\mathrm{M}$ & & DAKO & 100 & $50-100$ \\
\hline PCNA & M & & DAKO & 100 & $50-100$ \\
\hline PLAP & $\mathrm{P}$ & & DAKO & 100 & $25-50$ \\
\hline PAP & M & & Immunon & 50 & $50-100$ \\
\hline PSA & $\mathrm{M}$ & & Immunon & 20 & $20-40$ \\
\hline Prolactin & $\mathrm{P}$ & & DAKO & Neat & Neat \\
\hline $\mathrm{PP}$ & $\mathrm{P}$ & & DAKO & 900 & $600-900$ \\
\hline p53 & $\mathrm{M}$ & & DAKO & 100 & $50-100$ \\
\hline Progest Recep & $\mathrm{M}$ & & Immunotech & 50 & 50 \\
\hline Pre albumin & $\mathrm{P}$ & $\operatorname{Pr}$ & DAKO & 300 & 100 \\
\hline Sarcomeric actin & M & & DAKO & 40 & $10-40$ \\
\hline SMA & M & & DAKO & 100 & $50-100$ \\
\hline Secretin & $\mathrm{P}$ & & Bio Genex & Neat & Neat \\
\hline Serotonin & $\mathrm{M}$ & & DAKO & Neat & Neat \\
\hline SC & $\mathrm{P}$ & $\operatorname{Pr}$ & DAKO & 200 & $200-300$ \\
\hline Somatostatin & $\mathrm{P}$ & & DAKO & Neat & Neat \\
\hline S-100 & $\mathrm{P}$ & & DAKO & 400 & $400-800$ \\
\hline Synaptophysin & $\mathrm{P}$ & & DAKO & 100 & $20-50$ \\
\hline SAP-A & $\mathrm{M}$ & & DAKO & 100 & 100 \\
\hline ssDNA & $\mathrm{P}$ & & DAKO & 300 & $100-400$ \\
\hline TdT & $\mathrm{P}$ & $\mathrm{E}$ & DAKO & 40 & $10-40$ \\
\hline Thyroglobulin & M & & DAKO & 100 & $50-100$ \\
\hline TIA-1 & $\mathrm{M}$ & & Coulter & 1000 & ND \\
\hline $\mathrm{TM}$ & M & $\operatorname{Pr}$ & DAKO & 50 & $25-75$ \\
\hline TNF- $\alpha$ & $\mathrm{M}$ & & Beohringer & 20 & ND \\
\hline TSH & $\mathrm{P}$ & & DAKO & Neat & Neat \\
\hline TTF-1 & M & & DAKO & 100 & $100-200$ \\
\hline VIP & $\mathrm{P}$ & & Bio Genex & Neat & Neat \\
\hline Vimentin & M & & DAKO & 50 & $100-200$ \\
\hline Hercep Test & $\mathrm{P}$ & & DAKO & Kit & Kit \\
\hline
\end{tabular}

M, mouse monoclonal antibody; ND, no description; P, rabbit polyclonal antibody; G, goat polyclonal antibody; E, EDTA pretreatment; Pr, proteinase K pretreatment; Ur, urea pretreatment.

urea solution instead of TRS. Details for the primary antibodies are provided in Table 2.

\section{Discussion}

For the purpose of antigen retrieval, the efficacy of microwave pretreatment is well established. ${ }^{4,5,10-12}$ Advantages with microwave irradiation during antigen-antibody incubation were also reported recently, ${ }^{16}$ and we have concentrated attention on optimizing rapid immunostaining utilizing intermittent microwave irradiation (MI-77) during incubation with primary and secondary antibodies.

With early incubation procedures with intermittent microwave irradiation, for which specimens were directly put on a turn-table, ${ }^{17,18}$ only small numbers of the specimens could be processed at one time. To overcome this problem, we developed the special moist chamber, holding 10 specimens, which can be stacked inside the microwave equipment.

Comparison of the immunostaining between the standard method and our rapid protocol demonstrated equal or superior staining quality with the latter, especially with regard to nonspecific background staining. Microwaving causes minute vibrations more than 2.4 billion times $/ \mathrm{s},{ }^{19}$ which increases the probability of meeting of antibodies with specific antigens. At the same time, antibodies are easily dislodged from nonspecific binding sites by the motion.

From our 5 years experience, we conclude that the rapid method is appropriate for almost all primary antibodies used on a daily basis for pathological diagnosis at present. The benefits in terms of time are obvious. Indeed, rapid immunostaining methods applying for intraoperative frozen section diagnosis have been proposed by several institutes. ${ }^{20,21}$ Our method is also adaptable for practical use with frozen tissue sections omitting deparaffinizing and antigen retrieval. Thus, immunostaining with some antibodies may be achieved within $20 \mathrm{~min}$. We have preliminary confirmed that some epithelial markers (AE1/AE3, CAM 5.2), mesenchymal markers (vimentin, S100, ASMA), lymphoid markers (CD3, CD20, CD79a) and other diagnostic markers (GFAP, chromogranin A) demonstrate significant immunostaining with only $3 \mathrm{~min}$ irradiation (data not shown). 
a

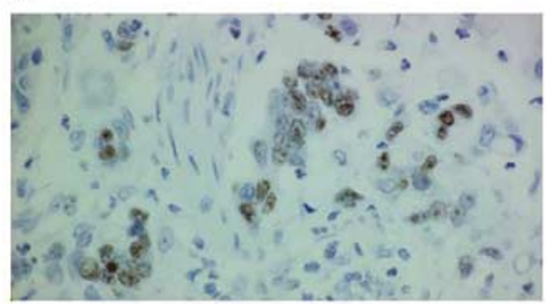

b
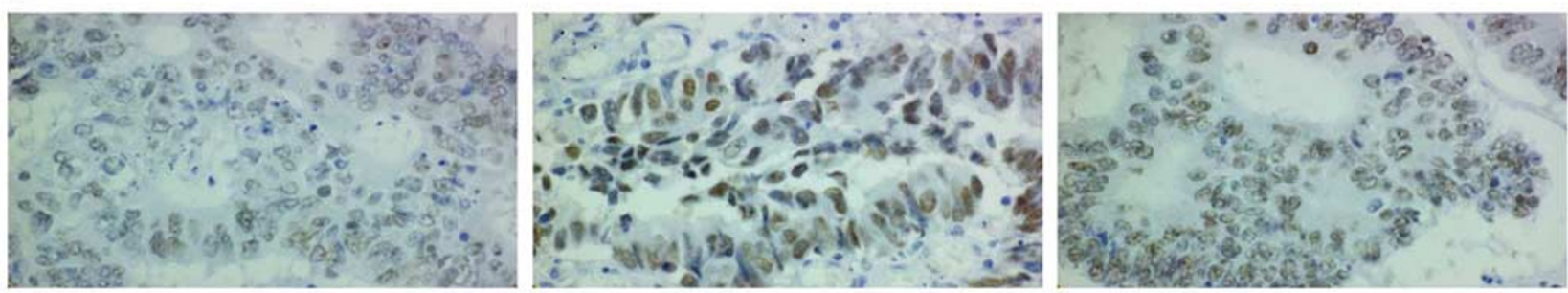

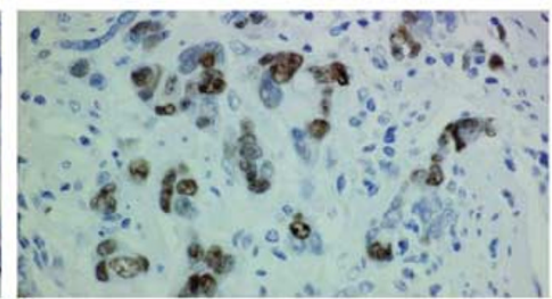

;

C
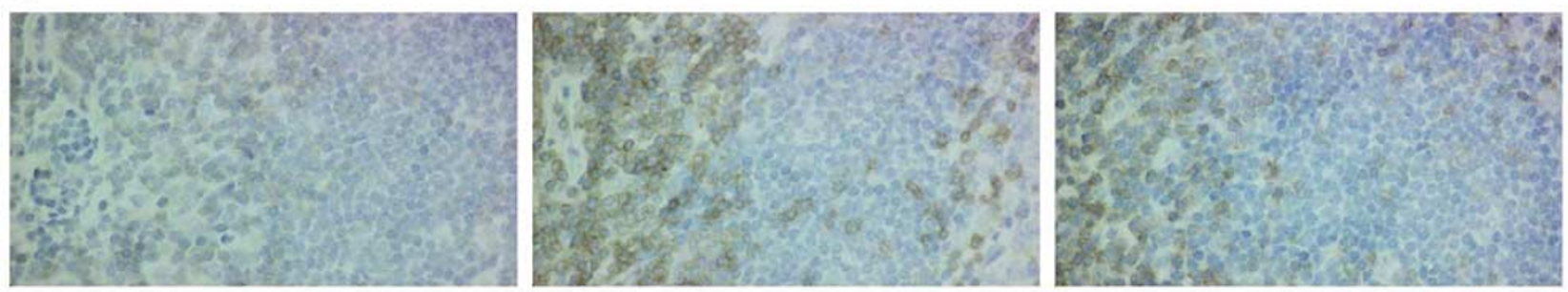

d
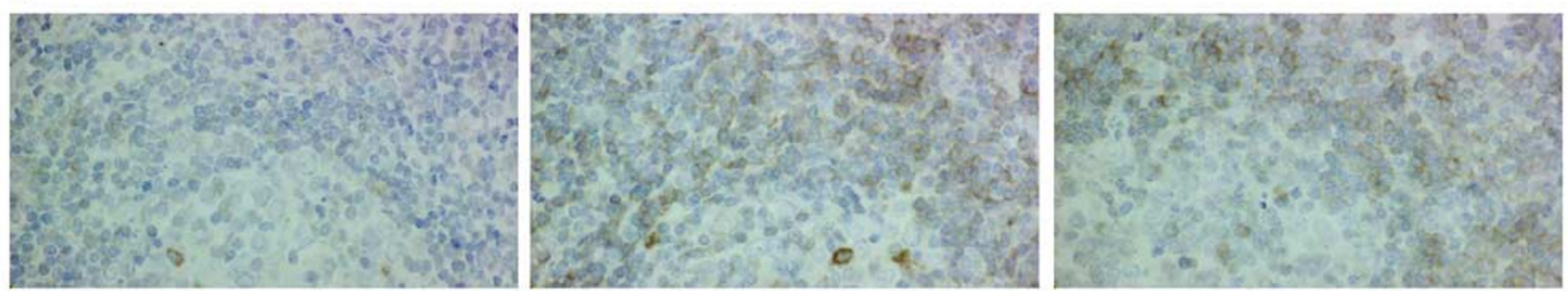

Figure 4 Comparison of staining quality among three different methods was shown in four different antibodies. Photomicrographs of a poorly differentiated colon adenocarcinoma incubated with an anti-MIB-1 antibody (a), of a moderately differentiated colon adenocarcinoma incubated with an anti-p53 antibody (b), of a lymph follicle with an anti-CD3 antibody (c) and anti-CD79a antibody (d) were shown. In each case, three figures were demonstrated. The left is the immunostaining of incubation in 10 min without microwaving, the center is that of incubation in $10 \mathrm{~min}$ with microwave irradiation (our rapid method), and the right is that of incubation in 60 min without microwaving (standard procedure). For all four antibodies, our rapid method (the center of each group) generated intense, frequent and significant staining, equal to or better than that with the standard procedure.

In conclusion, the presently described innovative immunostaining method combining special intermittent microwave equipment and moist chambers offers excellent staining quality for diagnosis while saving time and technical labor.

\section{Acknowledgement}

We specially thank Satoshi Ejiri, Chieko Kiya, Chizuko Murosaka and Kanako Nakagawa for their excellent technical supports. We also to thank Yukari Inoue for her secretarial assistance. This work was partially supported by the Japanese Ministry of Education, Science, Sports and Culture, Grant-inAid for Scientific Research 14770072 and 15922084 and the 21st century COE program in Japan.

\section{References}

1 Heitzmann H, Richards FM. Use of the avidin-biotin complex for specific staining of biological membranes in electron microscopy. Proc Natl Acad Sci USA 1974;71:3537-3541. 
2 Guesdon JL, Ternynck T, Avrameas S. The use of avidin-biotin interaction in immunoenzymatic techniques. J Histochem Cytochem 1979;27:1131-1139.

3 Hsu SM, Raine L, Fanger H. Use of avidin-biotinperoxidase complex (ABC) in immunoperoxidase techniques: a comparison between $\mathrm{ABC}$ and unlabeled antibody (PAP) procedures. J Histochem Cytochem 1981;29:577-580.

4 Wester K, Wahlund E, Sundstrom C, et al. Paraffin section storage and immunohistochemistry. Effects of time, temperature, fixation, and retrieval protocol with emphasis on p53 protein and MIB1 antigen. Appl Immunohistochem Mol Morphol 2000;8:61-70.

5 van den Broek LJ, van de Vijver MJ. Assessment of problems in diagnostic and research immunohistochemistry associated with epitome instability in stored paraffin sections. Appl Immunohistochem Mol Morphol 2000;8:316-321.

6 Frost AR, Sparks D, Grizzle WE. Methods of antigen recovery vary in their usefulness in unmasking specific antigens in immunohistochemistry. Appl Immunohistochem Mol Morphol 2000;8:236-243.

7 Kanai K, Nunoya T, Shibuya K, et al. Variations in effectiveness of antigen retrieval pretreatments for diagnostic immunohistochemistry. Res Vet Sci 1998; 64:57-61.

8 Imam SA, Young L, Chaiwun B, et al. Comparison of two microwave based antigen-retrieval solutions in unmasking epitopes in formalin-fixed tissue for immunostaining. Anticancer Res 1995;15:1153-1158.

9 Cattoretti G, Pileri S, Parravicini C, et al. Antigen unmasking on formalin-fixed, paraffin-embedded tissue sections. J Pathol 1993;171:83-98.

10 Leong AS. Microwaves in diagnostic immunohistochemistry. Eur J Morphol 1996;34:381-383.

11 Brown RW, Chirala R. Utility of microwave-citrate antigen retrieval in diagnostic immunohistochemistry. Mod Pathol 1995;8:515-520.
12 Cuevas EC, Bateman AC, Wilkins BS, et al. Microwave antigen retrieval in immunocytochemistry: a study of 80 antibodies. J Clin Pathol 1994;47: 448-452.

13 von Wasielewski R, Werner M, Nolte M, et al. Effects of antigen retrieval by microwave heating in formalinfixed tissue sections on a broad panel of antibodies. Histochemistry 1994;102:165-172.

14 Hoetelmans RW, van Slooten HJ, Keijzer R, et al. Comparison of the effects of microwave heating and high pressure cooking for antigen retrieval of human and rat Bc1-2 protein in formaldehyde-fixed, paraffinembedded sections. Biotech Histochem 2002;77: 137-144.

15 Taylor CR, Shi SR, Chen C, et al. Comparative study of antigen retrieval heating methods: microwave, microwave and pressure cooker, autoclave, and steamer. Biotech Histochem 1996;71:263-270.

16 Jackson P, Lalani EN, Boutsen J. Microwave-stimulated immunogold silver staining. Histochem J 1988;20: 353-358.

17 Hemmi A, Yamaguchi H, Seyama Y, et al. Study of microwave fixation using an immunohistochemical method. Rinsho Byori 1990;38:193-200 [in Japanese].

18 Douwes Dekker PB, Boon ME, Vardaxis NJ. Microwave immunoincubations using coverplate units. Eur J Morphol 1993;31:298-308.

19 Banik S, Bandyopadhyay S, Ganguly S. Bioeffects of microwave-a brief review. Bioresour Technol 2003; 87:155-159.

20 Chilosi M, Lestani M, Pedron S, et al. A rapid immunostaining method for frozen sections. Biotech Histochem 1994;69:235-239.

21 Nomoto S, Nakao A, Ichihara T, et al. Intraoperative quick immunoperoxidase staining: a useful adjunct to routine pathological diagnosis in pancreatic carcinoma. Hepatogastroenterology 1995;42:717-723. 